\title{
Frequency Domain Analysis of Tractor Tyre Enveloping Properties
}

\author{
B. STOJÍ́ \\ University of Novi Sad, Faculty of Technical Sciences, bstojic@unc.ac.rs
}

\begin{abstract}
Main source of vibration excitation for off-road vehicles are ground profile undulations. Most unprepared terrains are characterized by wavelength of unevenness that is of the order of magnitude of the contact length between tire and ground, so that, due to its shape and elasticity, tire actually behaves as geometric low-pass filter transforming real road profile geometry into effective vehicle vibration excitation. Since this effective profile represents real vehicle excitation, it is of interest to study this filtering behaviour in more depth. In this work, investigation of this kind of tire response has been studied for agricultural tractor tire rolling quasistatically over singular road obstacle. Frequency analysis of road excitation and tyre response was carried out in order to obtain their spectra and frequency response function magnitude of the tyre as filter was obtained by dividing input by output spectra. Final assessment of frequency response function magnitude was obtained by averaging instances obtained for different dimensions of input obstacles.
\end{abstract}

\section{Introduction}

Main source of vibration excitation for off-road vehicles are ground profile undulations. If tyre rolls over uneven ground whose irregularities have wavelength of the order of magnitude similar to tyre contact length, wheel centre will not follow exact ground geometry (as would be the case when using point-contact rigid follower instead of elastic tyre), but will travel along the path of different shape. If tyre motion happens in quasistatic manner, then tyre response (in view of the wheel centre trajectory shape) will have greater length and lower height compared to single road irregularity causing this response. This is the result of both tyre geometry and elasticity, and can be described as geometric low-pass filtering of the original road profile. Wheel centre trajectory is thereby considered effective road profile, since this motion represents real vehicle vibration excitation. This aspect of the tyre behaviour is referred to as "enveloping behaviour", since the tyre virtually envelopes i.e. wraps around ground irregularity of the short wavelength. Choosing quasistatic mode, it is possible to focus analysis purely on tyre enveloping behaviour, not taking into account tyre internal dynamics and vibration properties. Since this filtering effectively happens in frequency-domain, it can be of interest for indepth study of tyre enveloping response to analyse regarded phenomenon from such point of view. Goal of this work is to derive frequency response function of the tyre as filter, as basis for further study of this fundamental tractor vibration excitation mechanism. 


\section{Tyre geometric filtering mechanism}

Most unprepared terrains are characterized by wavelength of unevenness that is of the order of magnitude of the contact length between tyre and ground, so that, due to its shape and elasticity, tyre actually behaves as geometric low-pass filter transforming real road profile geometry into effective vehicle vibration excitation as shown in Figure 1.

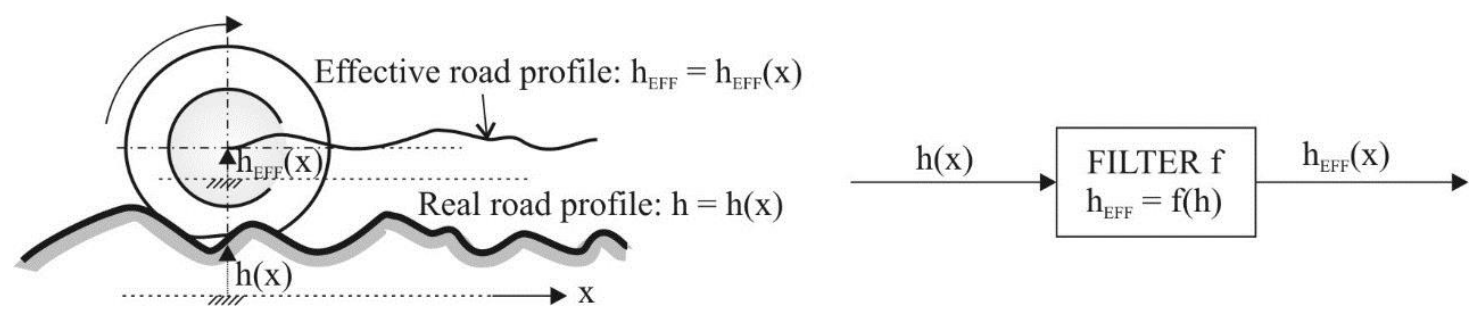

Figure 1Tyre as geometric low-pass filter for road undulations

Filtering mechanism is explained in more detail on the basis of Figure 2, according to [5]. Case (a) in the Figure 2 demonstrates the fact that, when rolling over singular road irregularity, leading segment of the tyre will come into contact with the outward edge of the irregularity before the middle point of the wheel is located above that edge. Due to the symmetry of the wheel in relation to the vertical axis, the same finding (but in the opposite direction) is also valid for the trailing segment of the tyre when coming down off the irregularity. This results in overall response curve of the tyre length being greater than the effective length of the rod irregularity, thus affecting frequency response towards lower frequency content. This part of the filtering mechanism is therefore based on geometrical properties.

The next factor of the mechanism of generating the tyre response is based on structural compliance as shown in Figure $3(b)$. When tyre rolls over the singular road irregularity whose length is smaller than the tyre contact length, the contact surface is deformed locally, partially or completely wrapping i.e. enveloping around the irregularity. This happens because of the tyre structure compliance, due to the action of the vertical load of the tyre. As a result, the maximum height of the tyre response curve remains smaller than the height of the unevenness itself, further contributing to the mitigation of response frequency spectrum magnitude.

Third aspect of the filtering mechanism is transformation of the sharp edges of the road irregularity to the smooth segments of the response curve, which is also kind of behaviour that corresponds to lowpass filter. This effect is though result of both mechanisms described previously, i.e. it is based both on the geometry and tyre elasticity. 


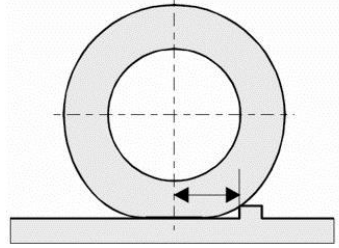

a)

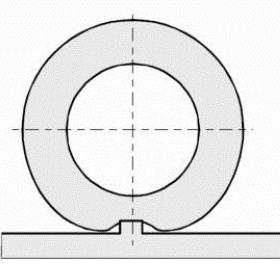

b)

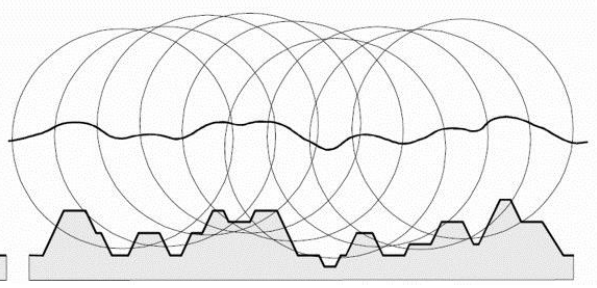

c)

Figure 2 Phenomena during tyre rolling over short-wavelength uneven terrain: a) increasing the response length compared to the excitation length; b) decreasing the response height compared to the excitation height; c) tyre behaviour resulting in geometrical filtering of the real road profile geometry [5]

Overall results of described mechanisms and effects in the spatial domain are higher wavelength (i.e., a lower spatial frequency) and smaller amplitude relative to the excitation. In other words, individual harmonics of the excitation frequency content are shifted toward lower frequencies in the response spectrum, at the same time being lowered in the magnitude. This low-pass filtering behaviour will be analysed in frequency domain subsequently.

\section{Experimental results and simulation model}

Since effective profile represents real vehicle excitation, it is of interest to study filtering behaviour of the tyre in more depth. In this work, investigation of this kind of tyre response has been studied for agricultural tractor tyre rolling quasistatically over uneven terrain. Experimental measurements required to obtain empirical data were conducted by using test facility based on the rail-guided cart in which tested wheel is mounted so that it can roll along the testing ground. Wheel thereby has freedom to move in vertical direction when negotiating road unevenness or obstacles, so that ground force remains constant if the quasi-static regime of motion is applied. Measuring vertical rim displacement vs. longitudinal wheel position, effective road profile curves are obtained. Test facility is described in more details in previous papers, e.g. [4].

Experimental investigations were carried out by quasi-static rolling of the tyre over the singular road irregularity in the form of rectangular obstacle. In sense of geometrical filtering, the process can be considered as rectangular pulse excitation passing through low-pass filter. Obstacles of different dimensions in view of height and length were used, as shown in the Table 1. Quasi-static conditions were adopted in order to be able to focus on the filtering behaviour and to exclude impact of all dynamic effects such as inertia, damping, load fluctuations etc. Dimensions of the tyre used for investigations were $12.4 \mathrm{R} 28$.

\begin{tabular}{|l|c|c|c|c|c|c|c|}
\hline Obstacle no. & 1 & 2 & 3 & 4 & 5 & 6 & 7 \\
\hline Length $L_{\text {obst }}[\mathrm{mm}]$ & 100 & 200 & 300 & 100 & 200 & 300 & 200 \\
\hline Height $H_{\text {obst }}[\mathrm{mm}]$ & 50 & 50 & 50 & 100 & 100 & 100 & 150 \\
\hline
\end{tabular}

Table 1. Dimensions of rectangular obstacles used in investigation

Typical qualitative shape of the response curve is depicted in the Figure 3. Curve represents the path of the wheel centre when tyre rolls quasistatically over rectangular road obstacle. This wheel motion introduces effective vibration excitation to the tractor. In the figure, geometrical parameters and 
relationship between input (which is rectangular obstacle) and output curve (wheel travel path) are also shown and explained.

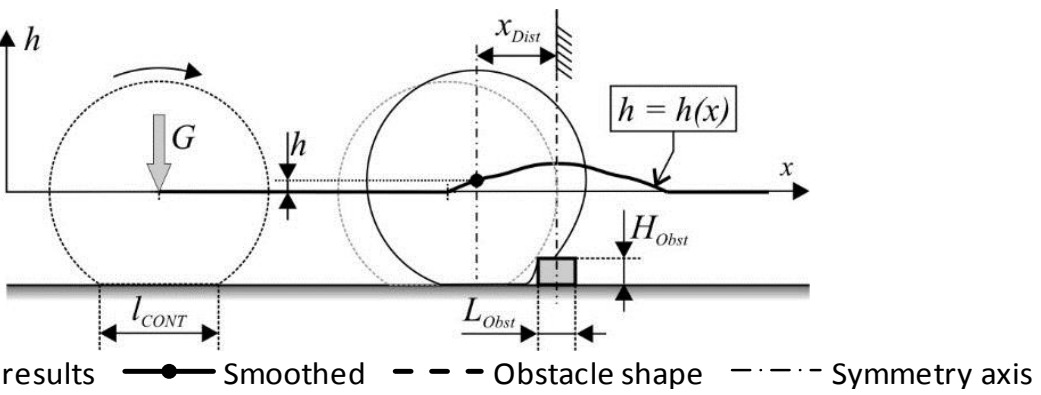

Figure 3 Example of the tyre enveloping curve for the singular ground obstacle of rectangular cross section: $h$ effective road profile height, $x$-longitudinal wheel travel, $h=h(x)$ - enveloping curve, $G$-vertical force exerted at the wheel, $l_{C O N T}$ - tyre contact length on the level ground, $x_{\text {Dist }}$ - wheel position with respect to the obstacle, $L_{\text {Obss }}, H_{O b s t}$ obstacle dimensions;

In some cases, intensive dissipation of measurement results was observed, sometimes not only in term of noise in data but also in shape of the curves obtained as shown in the Figure 4 (a). This phenomenon can be caused by uneven distribution of tyre radial stiffness, which can also be affected by stochastic position of lugs since it contributes to the bending stiffness of the tyre summit. In order to avoid problems connected with transformation to frequency domain of such set of experimental data, results obtained by using validated tyre model with radial and inter-radial springs are used instead because of their repeatability and unambiguousity. Schematic view of the model and comparison between model prediction and experimental results for one set of data are shown in Figure 4 (b) and (c) respectively.

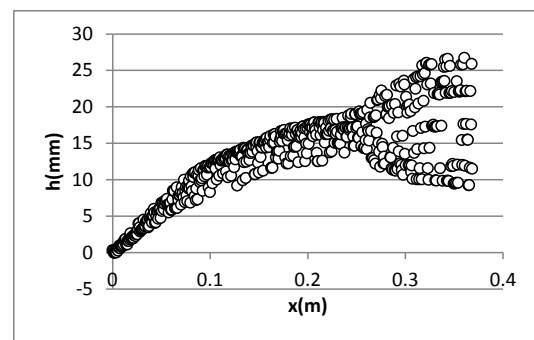

a)

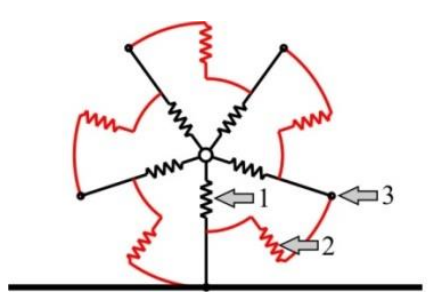

b)

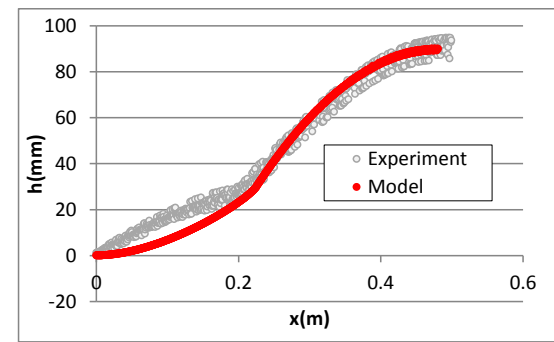

c)

Figure 4 a) Example of uncertainty of the response curve shape obtained experimentally; b) Schematic view of tyre model with radial and inter-radial springs used to simulate tyre behaviour; c) Comparison between model prediction and experimental results

Development and application of the tyre model with radial and inter-radial springs was based on existing approaches, e.g. [1][4], with adopting of some appropriate modifications and adaptations to the current field of application. Details about this model including its validation and application procedure are described in the paper Hiba! A hivatkozási forrás nem található.. 


\section{Frequency domain analysis}

Based on results obtained in spatial domain, firstly, frequency analysis of both excitation and response curves has been carried out. Regarding input, rectangular pulse is appropriate for analytical determination of the spectrum [3]:

$$
X(j \omega)=A \cdot \tau \cdot \frac{\sin (\omega \tau / 2)}{\omega \tau / 2}
$$

where in this case: $A=H_{\text {obst }}$ - pulse magnitude and $\tau=L_{\text {obst }}$ - pulse duration $\left(H_{\text {obst }}, L_{\text {obst }}\right.$ - obstacle dimensions according to the Figure 3 and Table 1).

Example of the input spectrum magnitude is shown in the Figure 5.

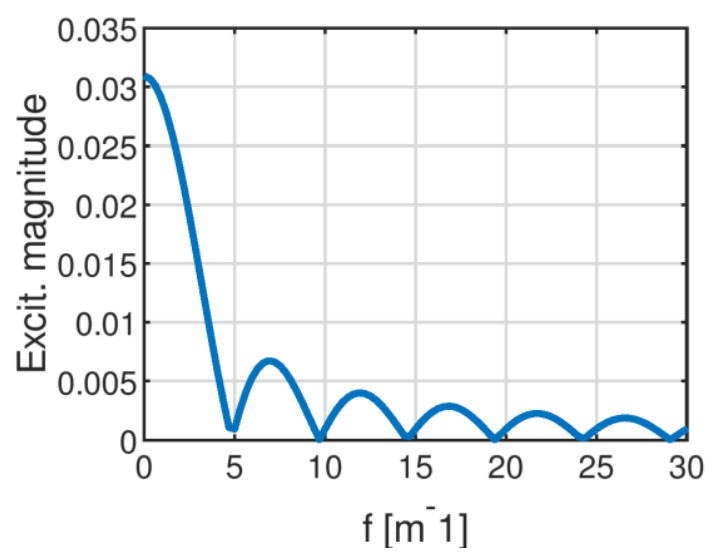

Figure 5 Magnitude of the input spectrum

Output spectra were obtained by using FFT function of the software Octave-Forge [1]. Example of the result is shown in the Figure 6 where intense damping of the spectrum at very low frequencies can be observed.

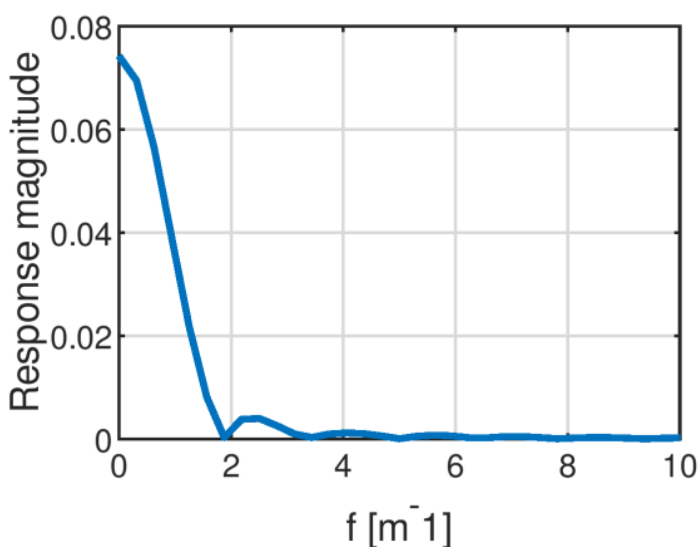

Figure 6 Magnitude of the output spectrum

Excitation spectrum was also obtained by using FFT algorithm and result was compared to analytical expression for single rectangular pulse in order to check whether adequate data sampling frequency was chosen. Since, by the nature of considered process, response is obtained by passing input signal through low-pass filter, this sampling frequency was also appropriate for output data. 
Spectra of both excitation and response, example of which is shown in Figures 5 and 6, were obtained for all 7 instances of experimental conditions according to Table 1. Further, instances of frequency response function magnitude curves were obtained dividing input by output spectra. A drawback of using rectangular excitation with this approach is having multiple zeros in excitation spectrum. Since distance between these zeros is proportional to pulse duration (i.e. to the length of the obstacle), attempt was made to compensate for this by using obstacles of different lengths and later account for this by averaging ratio between spectra of input and output. Therefore as final result, these curves were averaged to give approximate frequency response magnitude of the low-pass filter. Averaged frequency response function magnitude obtained this way is shown in the Figure 7.

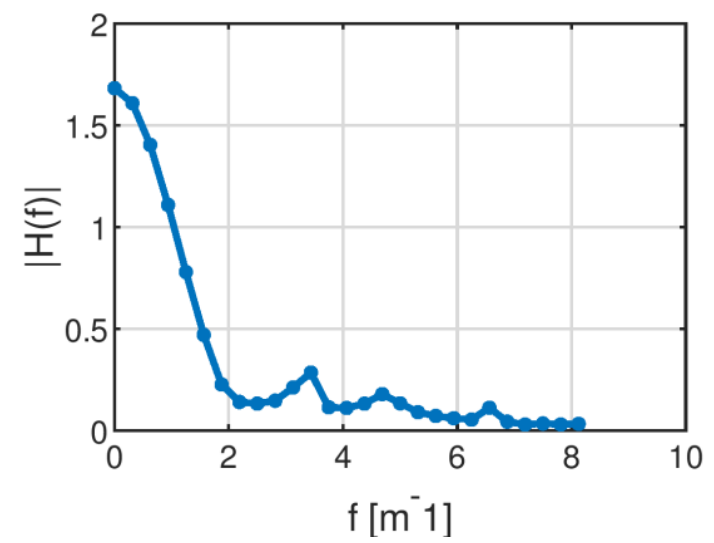

Figure 7 Averaged frequency response function magnitude

It can be seen that filter cut-off spatial frequency lies very low, somewhere between 2 and $6 \mathrm{~m}^{-1}$. Unevenness that can be observed in the curve shape could be consequence of numerical process of dividing small numbers since it is apparent that output spectrum magnitudes are all very low above $\sim 2 \div 3 \mathrm{~m}^{-1}$. Conclusion can be drawn that it is necessary to carry out more in-depth experimental study, obtaining more experimental data sets with broader range of parameter values, as well as to improve accuracy and precision of numerical data. That way it would be possible to determine some appropriate empirical mathematical function for frequency response. Moreover, this would enable final check if the properties of the tyre as dynamic system at all permit to define this quantity in proper manner, regarding above all system linearity.

\section{Conclusions}

In this paper frequency analysis of the response of the tyre rolling quasi-statically over singular road obstacle of rectangular shape was carried out. Tyre geometric low-pass filtering behaviour was in the focus of the study. Frequency response function magnitude of the filter was obtained by dividing input by output spectra. Using results for road obstacles of different dimensions it was attempted to overcome the drawback of having input spectrum with multiple zeros as it is the case for pulse excitation. Final assessment of frequency response function magnitude was obtained by averaging instances obtained individually. Resulting curve obtained this way exhibits uneven shape, which was attributed to numerical process of dividing small numbers since it is apparent that output spectrum magnitudes are all very low above $\sim 2 \div 3 \mathrm{~m}^{-1}$. It was concluded that it is necessary to carry out more in- 
depth experimental study, obtaining more experimental data sets with broader range of parameter values. Attempt to improve accuracy and precision of numerical data should also be made. That way it would be possible to determine some appropriate empirical mathematical function for frequency response. Furthermore, linearity of the system should be checked by classifying frequency response functions on the basis of different heights of input obstacles, which was omitted in the scope of the current study.

\section{References}

[1] J. M. Badalamenti - G. R. Doyle (1988) Radial-Interradial Spring Tire Models. Journal of Vibration. Acoustics, Stress and Reliability in Design, 110 (1) pp. 70-75

[2] J. W. Eaton - D. Bateman - S. Hauberg - R. Wehbring: GNU Octave version 4.2.2 manual: a highlevel interactive language for numerical computations, https://www.gnu.org/software/octave/doc/v4.2.2/, accessed 11.11.2018.

[3] M. Meyer (2006) Signalverarbeitung. Vieweg \& Sohn Verlag | GWV Fachverlage GmbH, Wiesbaden.

[4] B. Stojić - N. Poznanović - A. Poznić (2017) Research and Modeling of the Tractor Tire Enveloping Behavior. Journal of Vibration and Control. 23(2) pp. 290-304

[5] P. Witzel - S. Böttinger (2011) Upgrading of the Hohenheim Tyre Model to a radial approach for the simulation of obstacle passages. VDI Berichte Nr. 2124, VDI-Verlag, Düsseldorf. pp. 431-437.

[6] P. W. A. Zegelaar (1998) The Dynamic Response of Tyres to Brake Torque Variations and Road Unevennesses. PhD Thesis, Technical University of Delft, Netherlands, Delft, NL. 